\title{
Exact Solutions for Cosmological Models with a Scalar Field
}

\author{
H. Motavali ${ }^{1,2,3}$, M. Golshani ${ }^{3}$ [ \\ ${ }^{1}$ Department of Physics, McMaster University, Hamilton, Ontario, Canada L8S 4M1 \\ 2 Department of Physics, Amirkabir University of Technology, Tehran, Iran 15875-4413 \\ ${ }^{3}$ Institute for Studies in Theoretical Physics and Mathematics,Tehran, Iran 19395-1795
}

December 12, 2018

\begin{abstract}
We consider the existence of a Noether symmetry in the scalar-tensor theory of gravity in flat Friedman Robertson Walker (FRW) cosmology. The forms of coupling function $\omega(\phi)$ and generic potential $V(\phi)$ are obtained by requiring the existence of a Noether symmetry for such theory. We derive exact cosmological solutions of the field equations from a point-like Lagrangian.
\end{abstract}

\section{Introduction}

Scalar-tensor theories of gravity have been widely used in recent years. In principle, they are related to many fundamental theories as super-string theories, grand unified theories and quantum gravity. ${ }^{1,2}$ These theories allow the gravitational coupling to vary and act as a dynamical field..$^{3,4}$ In the Kaluza-Klein models this arises from a variation of the size of the internal dimensions. In a cosmological context such theories allow to look for dynamical answers for equations.

The general form of the extended gravitational actions in scalar-tensor theories can be written in terms of the Brans-Dicke field $\phi$ and the strength of the coupling between the scalar field and gravity which is represented by the dynamical coupling function $\omega(\phi)$. Furthermore, a nontrivial potential $V(\phi)$ may be introduced in terms of a scalar field which clearly affect the dynamics. Higher-order gravitational theories are equivalent to a scalar-tensor theory when $V(\phi)$ is non-zero but $\omega(\phi)=0$, which introduces Yukawa-type corrections to the Newtonian potential. ${ }^{5}$ The case of $V(\phi)=0$ ensures a strictly Newtonian weak field limit to lowest order. When the coupling functional $\omega(\phi)$ is considered to be a constant parameter, the scalar-tensor theory reduces to the Brans-Dicke theory.

In general, there is no unique method to determine the functional forms of $\omega(\phi)$ and $V(\phi)$. In this paper, we first prove the existence of the Noether symmetry and then we use this symmetry to find these functionals. The resulting functional forms for $\omega(\phi)$ and $V(\phi)$ are not independent

*e-mail address: motaval@theory.ipm.ac.ir

${ }^{\dagger}$ e-mail address: golshani@ihcs.ac.ir 
of each other. One may note that the Lagrangian in the action becomes point-like if we impose a flat Friedman Robertson Walker (FRW) metric.

The paper is organized as follows: the existence of the Noether symmetry is proved in section 2. In section 3 the exact solutions of the field equations are derived from a point-like Lagrangian for a quartic potential. The concluding remarks appear in the section 4 .

\section{Noether Symmetry}

We consider the action of scalar-tensor theory in the form

$$
S[\Phi]=\int d^{4} x \sqrt{-g}\left\{\Phi R+\frac{\omega(\Phi)}{\Phi} g^{\mu \nu} \nabla_{\mu} \Phi \nabla_{\nu} \Phi-V(\Phi)\right\}
$$

where $R$ is the scalar curvature, $\Phi$ denotes a real scalar field, non-minimally coupled to gravity, and $\omega(\Phi)$ and $V(\Phi)$ represent the coupling function and generic potential, respectively. The action (1) can be rewritten, with redefinition $\Phi=\phi^{2}$, as

$$
S[\phi]=\int d^{4} x \sqrt{-g}\left\{\phi^{2} R+4 \omega(\phi) g^{\mu \nu} \nabla_{\mu} \phi \nabla_{\nu} \phi-V(\phi)\right\} .
$$

In the cosmological case, when the space-time manifold is described by a flat FRW metric, the scalar curvature has the expression $R=-6\left(\ddot{a} / a+\dot{a}^{2} / a^{2}\right)$, in which $a$ is the scale factor of the universe and the dot denotes the derivative with respect to time. One can show simply that in this space-time manifold the Lagrangian density related to the action (2) takes the point-like form $^{6,7}$

$$
\mathcal{L}=12 a^{2} \dot{a} \phi \dot{\phi}+6 a \dot{a}^{2} \phi^{2}+a^{3}\left(4 \omega(\phi) \dot{\phi}^{2}-V(\phi)\right) .
$$

The corresponding Euler-Lagrange equations are given by

$$
\left[\frac{\ddot{a}}{a}+\frac{1}{2}\left(\frac{\dot{a}}{a}\right)^{2}\right] \phi^{2}+\left[\ddot{\phi}+2\left(\frac{\dot{a}}{a}\right) \dot{\phi}\right] \phi+\dot{\phi}^{2}-\left(\omega(\phi) \dot{\phi}^{2}-\frac{1}{4} V(\phi)\right)=0,
$$

and

$$
\dot{\phi} \omega(\phi)+3 \dot{\phi}\left(\frac{\dot{a}}{a}\right) \omega(\phi)+\frac{3}{2}\left[\frac{\ddot{a}}{a}+\left(\frac{\dot{a}}{a}\right)^{2}\right] \phi+\frac{1}{2}\left(\omega^{\prime}(\phi) \dot{\phi}^{2}+\frac{1}{4} V^{\prime}(\phi)\right)=0 .
$$

These equations are equivalent to the second order Einstein equation and the Klein-Gordon equation, in the flat FRW space-time, respectively.

We examine Noether symmetry of the Lagrangian (3) in order to determine the two unknown functions $\omega(\phi)$ and $V(\phi)$, as well as solving its dynamical field equations.

The infinitesimal generator of the Noether symmetry, namely the lift vector field $X$, is

$$
X=\alpha(\phi, a) \frac{\partial}{\partial a}+\beta(\phi, a) \frac{\partial}{\partial \phi}+\frac{d \alpha}{d t} \frac{\partial}{\partial \dot{a}}+\frac{d \beta}{d t} \frac{\partial}{\partial \dot{\phi}} .
$$

We try to determine $\alpha(\phi, a)$ and $\beta(\phi, a)$ by imposing the following condition ${ }^{8}$

$$
L_{X} \mathcal{L}=0,
$$


where $L_{X}$ is the Lie derivative with respect to the vector field $X$. Condition (6) leads to the following differential equations

$$
\begin{aligned}
& 3 \alpha V(\phi)+a \beta V^{\prime}(\phi)=0 \\
& 3 \alpha \omega(\phi)+\beta a \omega^{\prime}(\phi)+3 \phi \frac{\partial \alpha}{\partial \phi}+2 a \omega(\phi) \frac{\partial \beta}{\partial \phi}=0 \\
& \alpha \phi^{2}+2 \beta a \phi+2 a \phi^{2} \frac{\partial \alpha}{\partial a}+2 a^{2} \phi \frac{\partial \beta}{\partial a}=0 \\
& 2 \phi \alpha+a \beta+a \phi \frac{\partial \alpha}{\partial a}+\phi^{2} \frac{\partial \alpha}{\partial \phi}+a \phi \frac{\partial \beta}{\partial \phi}+\frac{2}{3} a^{2} \omega(\phi) \frac{\partial \beta}{\partial a}=0 .
\end{aligned}
$$

From Eq. (7) we have

$$
\alpha=-a \beta U(\phi)
$$

where we have used the definition

$$
U(\phi)=\frac{1}{3} \frac{V^{\prime}(\phi)}{V(\phi)}
$$

Substituting (11) into (8) and (9), we find that the variables $\phi$ and $a$ in $\beta(\phi, a)$ must separate as

$$
\beta(\phi, a)=f(\phi) a^{n}
$$

where the separation constant $n$ is given by

$$
n=\frac{\frac{3}{2} \phi U(\phi)-1}{1-\phi U(\phi)}
$$

and functional $f(\phi)$ has the following form

$$
f(\phi)=\exp \left\{\int \frac{\omega(\phi) U(\phi)+\phi U^{\prime}(\phi)-\frac{1}{3} \omega^{\prime}(\phi)}{\frac{2}{3} \omega(\phi)-\phi U(\phi)} d \phi\right\} .
$$

From the definition (12) and using (14) we obtain

$$
V(\phi)=\lambda \phi^{m}, \quad m=\frac{3(n+1)}{(n+3 / 2)}
$$

and

$$
U(\phi)=\frac{1}{3} m \phi^{-1}
$$

where $\lambda$ is an arbitrary integration constant. Putting these results into Eq. (10) one has

$$
(m-3) \phi \omega^{\prime}(\phi)+n \omega^{2}(\phi)-\left[m^{2}+m(1+4 n)-6\right] \omega(\phi)+4(n m+3 m-6) m=0 .
$$

This is a first-order differential equation for the dynamical coupling function $\omega(\phi)$ with the solution

$$
\omega(\phi)=k_{1}\left[\frac{\left(\phi / \phi_{0}\right)^{k_{2}}-1}{\left(\phi / \phi_{0}\right)^{k_{2}}+1}\right]+\omega_{0}
$$


where $\phi_{0}$ is an integration constant and $k_{1}, k_{2}$ and $\omega_{0}$ are defined as

$$
k_{1}=\frac{3}{(2 n+3)^{2}}, \quad k_{2}=\frac{-2 n}{2 n+3}, \quad \omega_{0}=\frac{3\left(8 n^{2}+24 n+17\right)}{(2 n+3)^{2}} .
$$

Substituting (17) and (19) into (15) and using (13) and (11), one gets

$$
\alpha(\phi, a)=-\frac{1}{3} \beta_{0} m \phi_{0}^{-1} a^{n}\left(\phi / \phi_{0}\right)^{l_{1}-1}\left[\left(\phi / \phi_{0}\right)^{k_{2}}-\xi\right]^{l_{2}}\left[\left(\phi / \phi_{0}\right)^{k_{2}}+1\right]^{l_{3}}
$$

and

$$
\beta(\phi, a)=\beta_{0} a^{n}\left(\phi / \phi_{0}\right)^{l_{1}}\left[\left(\phi / \phi_{0}\right)^{k_{2}}-\xi\right]^{l_{2}}\left[\left(\phi / \phi_{0}\right)^{k_{2}}+1\right]^{l_{3}},
$$

where $\beta_{0}$ is an integration constant, and we have used the definitions

$$
\begin{aligned}
& l_{1}=\frac{m\left(\omega_{0}-k_{1}-1\right)}{2 \omega_{0}-2 k_{1}-m} \\
& l_{2}=\frac{2 k_{1}^{2} k_{2}-k_{2}\left(m-2 \omega_{0}\right)^{2} / 2-2 m(m-2)}{4 k_{1}^{2}-\left(m-2 \omega_{0}\right)^{2}} \\
& l_{3}=-\frac{2 k_{1}^{2} k_{2}-k_{2}\left(m-2 \omega_{0}\right)^{2} / 2-2 m(m-2)^{2}\left(k_{1}+1\right)}{4 k_{1}^{2}-\left(m-2 \omega_{0}\right)^{2}} \\
& \xi=\frac{2 k_{1}-\left(m-2 \omega_{0}\right)}{2 k_{1}+\left(m-2 \omega_{0}\right)} .
\end{aligned}
$$

Therefore, the vector field $X$ exists, and the existence of the Noether symmetry leads to the determination of the class of potentials (16) and the dynamical coupling function (19), for arbitrary values of $n$, except $n=-3 / 2$. In principle, the existence of the Noether symmetry means that there must exist a constant of motion. In this case one may compute it using the Cartan one-form associated with the Lagrangian (3)

$$
\theta_{\mathcal{L}} \equiv \frac{\partial \mathcal{L}}{\partial \dot{a}} d a+\frac{\partial \mathcal{L}}{\partial \dot{\phi}} d \phi
$$

Contracting $\theta_{\mathcal{L}}$ with $X$ gives the following required constant of motion

$$
i_{X} \theta_{\mathcal{L}}=12 a \phi \alpha(\phi, a)\{a \dot{\phi}+\phi \dot{a}\}+4 a^{2} \beta(\phi, a)\{3 \dot{a} \phi+2 a \dot{\phi} \omega(\phi)\}
$$

where $\omega(\phi), \alpha(\phi, a)$ and $\beta(\phi, a)$ are given by (19), (20) and (21), respectively.

\section{Dynamical Field Equations and Solutions}

Scalar-tensor theory reduces to Brans-Dicke theory when the coupling function $\omega(\phi)$ is taken to be a constant. For mathematical simplicity, we analyze the solutions of the field equations (4) and (5) for $n=-3$ and in the case that $\omega(\phi)$ is a constant parameter such as $\omega$. In this case the Lagrangian (3) takes the form

$$
\mathcal{L}=12 \dot{a} a^{2} \phi \dot{\phi}+6 \dot{a}^{2} a \phi^{2}+a^{3}\left(4 \omega \dot{\phi}^{2}-\lambda \phi^{4}\right) .
$$

The corresponding field equations are given by

$$
\left[\frac{\ddot{a}}{a}+\frac{1}{2}\left(\frac{\dot{a}}{a}\right)^{2}\right] \phi^{2}+\left[\ddot{\phi}+2\left(\frac{\dot{a}}{a}\right) \dot{\phi}\right] \phi+\dot{\phi}^{2}+\frac{1}{4} \lambda \phi^{4}=0
$$


and

$$
\omega \ddot{\phi}+3 \omega \dot{\phi}\left(\frac{\dot{a}}{a}\right)+\frac{3}{2}\left[\frac{\ddot{a}}{a}+\left(\frac{\dot{a}}{a}\right)^{2}\right] \phi+\frac{1}{2} \lambda \phi^{3}=0 .
$$

One may select the initial conditions of the field equations (23) and (24) such that the energy function associated with the Lagrangian (22) vanishes

$$
\begin{aligned}
E_{\mathcal{L}} & =\frac{\partial \mathcal{L}}{\partial \dot{a}} \dot{a}+\frac{\partial \mathcal{L}}{\partial \dot{\phi}} \dot{\phi}-\mathcal{L} \\
& =12 \dot{a} a^{2} \phi \dot{\phi}+6 \dot{a}^{2} a \phi^{2}+a^{3}\left(4 \omega \dot{\phi}^{2}+\lambda \phi^{4}\right)=0 .
\end{aligned}
$$

In fact, by choosing $E_{\mathcal{L}}=0$, one imposes a constraint on the integration constants. From (20) and (21) we have

$$
\alpha=-\frac{4}{3} \frac{\beta_{0}}{a^{2} \phi^{2}}, \quad \beta=\frac{\beta_{0}}{a^{3} \phi}
$$

for $n=-3$. Now, the existence of the vector field $X$ can be used to find a non-degenerate point transformation

$$
(a, \phi) \longrightarrow(p, q)
$$

such that the transformed Lagrangian is cyclic in one of the new variables. A possible way is to compute the Cartan one-form associated with Lagrangian $\mathcal{L}$, namely

$$
\left\{\begin{array}{l}
i_{X}(d p)=1 \\
i_{X}(d q)=0
\end{array}\right.
$$

or explicitly

$$
\left\{\begin{array}{l}
\alpha \frac{\partial p}{\partial a}+\beta \frac{\partial p}{\partial \phi}=1 \\
\alpha \frac{\partial q}{\partial a}+\beta \frac{\partial q}{\partial \phi}=0
\end{array}\right.
$$

Substituting (26) into (27), yields the solutions

$$
\left\{\begin{array}{c}
p=-\frac{1}{2 \beta_{0}} a^{3} \phi^{2} \\
q=\gamma_{0} a^{3 / \gamma} \phi^{4 / \gamma}
\end{array}\right.
$$

where $\gamma_{0}$ and $\gamma$ are constant. Under these transformations, the Lagrangian (22) takes the form

$$
\mathcal{L}=2 \beta_{0}\left\{2 \gamma(\omega-5 / 3) \frac{\dot{p} \dot{q}}{q}+(\omega-4 / 3)\left(\frac{\dot{p}^{2}}{p}+\gamma^{2} \frac{p \dot{q}^{2}}{q^{2}}\right)+\frac{\lambda}{2 \beta_{0} \gamma_{0}} q^{\gamma}\right\} .
$$

One can easily check that Eq. (18) has two solutions: $\omega=3 / 2$ and $\omega=4 / 3$ for $n=-3$. Now, if one computes the Hessian determinant $\mathcal{H}=\left|\partial^{2} \mathcal{L} / \partial \dot{\phi} \partial \dot{a}\right|$, one finds that $\omega=3 / 2$ leads to the degeneration of $\mathcal{L}$. In other words, this value of $\omega$ does not allow a Hamiltonian formulation for the theory and leads to pathological dynamics. Thus, one can disregard $\omega=3 / 2$. For $\omega=4 / 3$ the second term of (29) disappears and $\mathcal{L}$ takes the form

$$
\mathcal{L}=-\frac{4}{3} \beta_{0} \gamma \frac{\dot{p} \dot{q}}{q}+\frac{\lambda}{\gamma_{0}} q^{\gamma} .
$$


This Lagrangian clearly does not depend on $p$. Therefore, in new configuration space $(p, q)$, the variable $p$ is cyclic. This implies the existence of the Noether symmetry.

The corresponding field equations for the last Lagrangian are given by

$$
\ddot{p}+\frac{3 \lambda}{4 \beta_{0} \gamma_{0}} q^{\gamma}=0
$$

and

$$
\dot{q}-r_{0} q=0
$$

where $r_{0}$ is constant of motion. The solutions of these equations are

$$
p=s_{0}\left(e^{r_{0} \gamma t}+p_{0}\right)+\dot{p_{0}} t
$$

and

$$
q=q_{0} e^{r_{0} t}
$$

where $p_{0}, \dot{p_{0}}$ and $q_{0}$ are integration constants and $s_{0}$ is given by

$$
s_{0}=-\frac{3 \lambda q_{0}^{\gamma}}{4 \beta_{0} \gamma_{0} \gamma^{2} r_{0}^{2}} \text {. }
$$

In new configuration space $(p, q)$, the condition $(25)$ can be rewritten as

$$
E_{\mathcal{L}}=-\frac{4}{3} \beta_{0} \gamma \frac{\dot{p} \dot{q}}{q}-\frac{\lambda}{\gamma_{0}} q^{\gamma}=0 .
$$

Substituting (31) and (32) into (33), leads to $\dot{p_{0}}=0$, and so the solution (31) reduces to

$$
p=s_{0}\left(e^{r_{0} \gamma t}+p_{0}\right) .
$$

Putting (32) and (34) into (28), one gets the following solutions

$$
\phi=\psi_{0} e^{r_{0} \gamma t / 2}\left(e^{r_{0} \gamma t}+p_{0}\right)^{-1 / 2}, \quad \psi_{0}=\left(-\frac{q_{0}^{\gamma}}{2 s_{0} \beta_{0} \gamma_{0}^{\gamma}}\right)^{1 / 2}
$$

and

$$
a=a_{0} e^{-r_{0} \gamma t}\left(e^{r_{0} \gamma t}+p_{0}\right)^{2 / 3}, \quad a_{0}=\left(\frac{4 s_{0}^{2} \beta_{0}^{2} \gamma_{0}^{\gamma}}{q_{0}^{\gamma}}\right)^{1 / 3} .
$$

In the limit $t \rightarrow \infty$ the scalar field $\phi$ approaches a constant value $\psi_{0}$, and then the Einstein gravity is recovered and the Newtonian gravitational constant is identified as $G_{N}={\frac{1}{\psi_{0}}}^{2}$.

\section{Concluding Remarks}

We have studied scalar-tensor theories of gravity in which the coupling function $\omega(\phi)$ and the generic potential $V(\phi)$ are unknown. The form of the coupling function and the potential are determined using Noether symmetry in a flat FRW background. In the special case $n=-3$, we showed that Noether symmetry exists for $\omega=3 / 4$, and we derived the corresponding constant of motion, $r_{0}$. Furthermore, the exact solutions of the field equations were derived from a point-like Lagrangian for a quartic potential. It is also shown that in the case of $\omega=3 / 2$, there is no Noether symmetry for the Lagrangian. However, it is interesting to note that the theory is exactly conformal invariant. ${ }^{9}$ Further attempts seems to be necessary to understand this relationship more deeply. 


\section{References}

[1] A. Zee, Phys. Rev. Lett. 42, 417 (1979);

L. Smolin, Nucl. Phys. B 160, 253 (1979);

S. Adler, Phys. Rev. Lett. 44, 1567 (1980).

[2] S. Capozziello and R. De Ritis Phys. Lett. A177, 1 (1993).

[3] P. Jordan, Z. Phys. 157, 112 (1959).

[4] C. Brans and R. H. Dicke, Phys. Rev. 124, 925 (1961).

[5] P. Teyssandier and P. Tourrence, J. Math. Phys. 24, 2793 (1983);

D. Wands, Class. Quantum Grav. 7, 269 (1994).

[6] R. De Ritis, G. Platania, P. Scudellaro, and C. Stornaiolo, Gen. Relativ. Gravit. 22, 97 (1990).

[7] S. Capozziello, R. De Ritis, C. Rubano and P. Scudellaro Riv, Nuovo Cimento 4 (1996).

[8] R. Abraham and J. Marsden 1978 Foundation of Mechanics (New York; Benjamin)

G. Marmo, E. J. Saletan, A. Simoni and B. Vitale 1985 Dynamical Systems.

A Differential Geometric Approach to Symmetry and Reduction (New York: Wiley).

[9] H. Motavali, H. Salehi and M. Golshani, Int. J. Mod. Phys. A15, 983 (2000).

H. Motavali, H. Salehi and M. Golshani, Int. J. Mod. Phys. Lett. A14, 2481 (1999). 